\title{
Mass Dampers with Limited Amplitude
}

\author{
Laust Tophøj \& Nikolaj Grathwol \\ TG Dampers ApS, Valdemarshaab 11, 4600 Køge, Denmark \\ contact: lt@tgdampers.dk
}

\begin{abstract}
Mass dampers are widely used in engineering applications. We consider the effects of limitations on the damper amplitude.

Using simple methods to analyze very general mass dampers, we find an upper limit to the damping. The maximum damping logarithmic decrement is $\delta_{\max }=4 \mu \alpha$, where $\mu$ is the mass ratio, and $\alpha$ is the amplitude ratio of damper to structure amplitude.

The result is further discussed in relation to Tuned Mass Dampers (TMDs), which can perform very well if there is enough avaliable space. In practice, amplitude limits always apply, and our result can be used to relate these to the damper performance.

Our result also applies to active devices, which have to obey the limit mentioned above.

Simulated tests of TMDs and other mass dampers are described. The damping is measured both by decay tests and by forced motion test. The methods agree well in the amplitude-limited regime. In other cases, decay tests are difficulet to interpret, indicating that one needs to be very careful when measuring damping of 2DOF systems based solely on decay tests.

We hope that our result may inform the selection and design of mass dampers in the future, where one should consider amplitude limits as the very first step.
\end{abstract}

\section{Introduction}

Many structures are equipped with a mass damper, see e.g. Fig. 1. A typical design process for such dampers sees much time spent on tuning dampers for optimal behaviour in a linear sence. The limited available space is often considered more as an afterthought. But the space-limitations fundamentally restrict the performance. Below we present an extremely simple result, which limits the damping provided by any mass damper. It is our hope that the result can serve as a framework for practical damper design in the future.

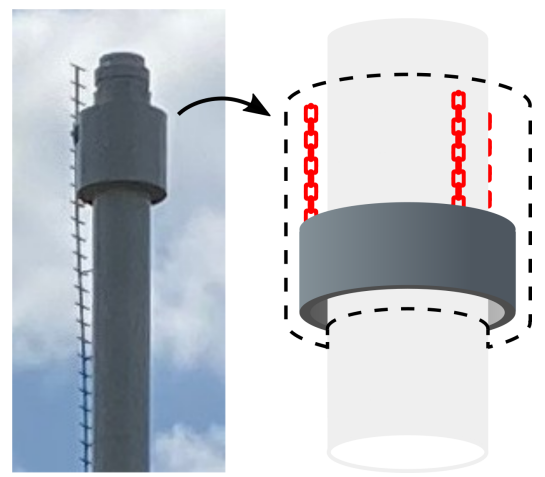

Figure 1: Steel stack with a Tuned Mass Damper (TMD). Cf. Fig. 2. This type of damper is very common on steel stacks. The damper layout is illustrated to the right. A heavy steel ring pendulum hangs from a number of chains (red), contained in a narrow annular chamber mounted on the outside of the stack. The width of the annular chamber determines the maximum movement of the pendulum. We shall show that this movement limit is extremely important to the damper performance.
Damping vibrations is critical in engineering applications such as civil engineering, industrial production, transportation and energy production, e.g. in wind turbines. Uncontrolled vibrations can harm structures and equipment and pose both danger and annoyance to humans.

A popular way to damp vibrations is to install a mass damper, i.e. a relatively small and light device installed on the structure. The mass damper is a local device, only connected to the structure at one location, so that mechanical connections to the ground (e.g. guy-wires) are avoided. Instead the mass damper works by exchanging inertial forces with the structure. One might think of the mass damper as holding on to a local inertial frame rather than to the ground. This property allows mass dampers to be used at inaccessible locations and even in moving ships, aircraft and other vehicles.

We shall deviate from the significant litterature on mass dampers, which mainly deals with specific types of the above dampers, and focus on very general properties of mass dampers, which apply to all types of mass dampers. In particular, we investigate the maximum damping, which a mass damper of finite size can deliver. The analysis only uses elementary methods but still yield results, which to our knowledge have not been reported previously.

\subsection{Example}

A steel stack of height $25 \mathrm{~m}$ and diameter $1 \mathrm{~m}$ is vulnerable to swaying Vortex Induced Vibrations (VIV). A Tuned Mass Damper (or TMD) [1] is installed near the stack top, see Fig. 1. The TMD is a heavy pendulum attached to the 
stack, partially immersed in a viscous liquid (not shown). Note that the subsequent analysis applies to all types of mass dampers, see Fig. 2 and Sec. 1.2.

The effective (modal) mass of the stack is $m_{0}=$ $5000 \mathrm{~kg}$, and the TMD mass is $m_{1}=100 \mathrm{~kg}$, so the mass ratio is $\mu=m_{1} / m_{0}=2 \%$. The damper is restricted to move within a chamber of width $0.4 \mathrm{~m}$, so the maximum damper amplitude is approximately $a_{1}=0.2 \mathrm{~m}$. Problematic vibrations of the steel stack of typical amplitude $a_{0}=0.5 \mathrm{~m}$ (see [2]) are to be prevented by the TMD. The ratio between the amplitudes is $\alpha=a_{1} / a_{0}=0.4$. Using a common rule-of-thumb for the performance of a TMD, see Sec. 2.3 and (22), one would expect the optimal TMD to deliver a damping of approximately $\delta_{\text {TMD opt. }}=2.2 \cdot \sqrt{\mu}=31 \%$. However, using our result below, (13), we see that the maximum damping due to the damper given the restricted space is

$$
\delta_{\max }=4 \cdot \mu \cdot \alpha=3 \% \approx \frac{\delta_{T M D, o p t} .}{10} .
$$

Insufficient space for the damper motion is a common flaw in the design of TMDs, and we know from our own experience that this is the cause behind several dramatic cases of problematic stack vibrations. It is our recommendation that one starts by considering the available space, using (13), when selecting and designing any mass damper.

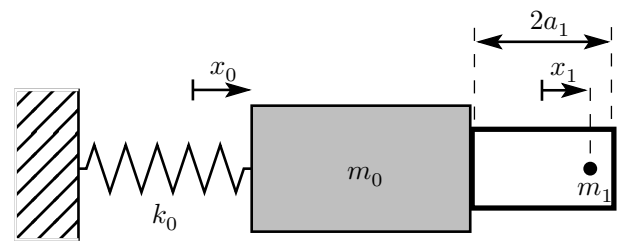

Figure 2: Sketch of a structure $m_{0}$ whose position $x_{0}$ vibrates with the amplitude $a_{0}$. A point-like damper mass $m_{1}$ is constrained within a finite container fixed to the structure, such that its position $x_{1}$ relative to the structure is $-a_{1} \leq x_{1} \leq a_{1}$. This sketch represents any type of mass damper, see e.g. Fig. 1 .

\subsection{Background}

Vibration control methods have wide applications, and have been discussed at great length in the litterature. see e.g. $[1,3,4]$, or the more modern and elementary $[5,6]$. Much work has focused on the linear systems behaviour of damping systems, such as TMDs, e.g. $[7,8,9,10,11,12,13]$, and many works on active dampers, $[4,14,15]$. However, these work generally focus on tuning aspects and on the mathematics of control theory. Other works present theory on impact dampers / pounding dampers [16, 17, 18, 19, 20, 21], particle dampers [22, 23]. For a more detailed discussion of impact dynamics and stability of periodic solutions, see e.g. [21, 24, 25, 26]. Tuned Liquid Dampers (TLDs) / sloshing dampers [27, 28]. Tuned Liquid Column Dampers (TLCDs), [29].

The fact that any real damper must be finite with a limited damper amplitude and the associated performance limits seem to have been somewhat neglected.

We shall focus on very general properties of mass dampers, relating to the conservation of momentum of the entire damper-structure system. The results apply to mass dampers of any type.

\subsection{Methods and assumptions}

The main analysis of this paper is done using the approximation that the damper acts as a small perturbation on the structure motion.

This approximation is excellent, as long as the mass ratio $\mu=m_{1} / m_{0}$ of the damper mass $m_{1}$ to the structure mass $m_{0}$ is small. As a rule-of-thumb, the results are useful up to about $\mu \lesssim 10 \%$. We furthermore ignore some aspects of the spectral character of the driving forces. In particular, we consider motion at the structure eigenfrequency. This too remains a good approximation for small values of $\mu \lesssim 10 \%$. We restrict our attention to periodic motions of the damper, ignoring possible non-unique motions and chaos. These topics are covered elsewhere, see e.g. $[21,24,25]$. We will often describe the structure motion as an undisturbed sine motion, although much of the analysis remains valid for any periodic structure motion, see below.

In a few cases, we will work outside the abovementioned framework of undisturbed harmonic structure motion and consider the free decay of structures with an attached mass damper. The results of the two methods agree well.

We have kept the paper deliberately short and simple and focused on the upper limit on the damping of any finite mass damper.

\section{Analysis}

Below, we use concepts common to basic theory of vibrations and vibration damping, see [6].

Consider the structure shown in Fig. 2. The structure of mass $m_{0}$ moves according to $x_{0}=x_{0}(t)$, and the position of the damper centre-of-mass relative to $x_{0}$ is $x_{1}=x_{1}(t){ }^{1}$

Both $x_{0}(t)$ and $x_{1}(t)$ are considered periodic with period $T$. We introduce the angular frequency $\omega \equiv \frac{2 \pi}{T}$ and the non-dimensional time $\tau=\omega t$. Then

$$
\begin{aligned}
& x_{0}(t)=a_{0} \xi_{0}(\tau)=a_{0} \cos (\omega t) . \\
& x_{1}(t)=a_{1} \xi_{1}(\tau) .
\end{aligned}
$$

where we have introduced the amplitudes $a_{0}$ and $a_{1}$, such that $-1 \leq \xi_{0} \leq 1$ and $-1 \leq \xi_{1} \leq 1$.

\footnotetext{
${ }^{1}$ We shall write $x_{0}(t)$ as a cosine function of time, but note that the results below, in particular (8) would hold for any bounded periodic functions $x_{0}(t)$ and $x_{1}(t)$. We furthermore consider the amplitudes $a_{0}$ and $a_{1}$ as constant quantities, though in reality they may be slowly drifting. The damping measured during constant amplitude vibrations (considering how much energy is needed to sustain the vibrations) is approximately equal to the damping measured during free decays of the structure. This is a good approximation for $\mu \lesssim 10 \%$.
} 
The mechanical energy associated with (2) is given by

$$
E_{0}=\frac{1}{2} m_{0} a_{0}^{2} \omega^{2} .
$$

Let a mass damper consist of a mass $m_{1}$ whose position is $x_{1}=x_{1}(t)$. The damper may be composed of many mass parts or a volume of dense liquid, in which case $x_{1}$ describes the position of the centre-of-mass. Alternatively, the damper may be simple as sketched in Fig. 2. The damper generates a reaction force $F$ on the structure with $F$ given from Newton's second law as

$$
F=-m_{1}\left(\ddot{x}_{0}+\ddot{x}_{1}\right)
$$

Damping is typically measured using the logarithmic decrement $\delta$, which is defined in terms of the decaying vibrations of a structure released from an initial displacement as

$$
\delta \equiv \log \frac{a_{0,}}{a_{0, \text { after }}} .
$$

Here, log is the natural logarithm, $a_{0}$ is the peak amplitude at a given time, and $a_{0, \text { after }}$ is the peak amplitude one oscillation cycle later. Using (4), we write

$$
\delta=\log \sqrt{\frac{E_{0}}{E_{0, \text { after }}}} \approx \frac{1}{2} \frac{\Delta E}{E_{0}},
$$

where we have expanded to lowest order in $\Delta E / E_{0}$, and $\Delta E$ is the energy dissipated during one oscillation cycle:

$$
\begin{aligned}
\Delta E & =-\int_{0}^{T} \dot{x}_{0} F \mathrm{~d} t=m_{1} \int_{0}^{T}\left(\dot{x}_{0} \ddot{x}_{1}+\dot{x}_{0} \ddot{x}_{0}\right) \mathrm{d} t . \\
& =m_{1} a_{0} a_{1} \omega^{2} \int_{0}^{2 \pi} \xi_{0}^{\prime}(\tau) \xi_{1}^{\prime \prime}(\tau) \mathrm{d} \tau,
\end{aligned}
$$

where we have used the periodicity of $x_{0}$ to get rid of the integral over $\dot{x}_{0} \ddot{x}_{0}=\frac{1}{2} \frac{\mathrm{d}}{\mathrm{d} t}\left(\dot{x}_{0}^{2}\right)$ and changed the integration variable to $\tau$.

Inserting in (7), we get

$$
\begin{aligned}
\delta & =\frac{1}{2} \frac{m_{1} a_{0} a_{1} \omega^{2} \int_{0}^{2 \pi} \xi_{0}^{\prime} \xi_{1}^{\prime \prime} \mathrm{d} \tau}{\frac{1}{2} m_{0} a_{0}^{2} \omega^{2}} \\
& =\frac{m_{1}}{m_{0}} \frac{a_{1}}{a_{0}} \int_{0}^{2 \pi} \xi_{0}^{\prime} \xi_{1}^{\prime \prime} \mathrm{d} \tau \\
& =\frac{m_{1}}{m_{0}} \frac{a_{1}}{a_{0}} \int_{0}^{2 \pi} \xi_{0}^{\prime \prime \prime} \xi_{1} \mathrm{~d} \tau \\
& =\mu \alpha I .
\end{aligned}
$$

Where $\mu \equiv \frac{m_{1}}{m_{0}}$ is the damper mass ratio, and $\alpha \equiv \frac{a_{1}}{a_{0}}$ is the damper amplitude ratio (which is sometimes vaguely referred to in the engineering community as the Dynamic Amplification Ratio or DAF). In the third line of (9), we have used integration by parts and exploited that $\xi_{0}(\tau)$, $\xi_{1}(\tau)$ as well as their derivatives are $2 \pi$-periodic functions of $\tau$ to rewrite the integral (which we will refer to as $I$ ) as

$$
I \equiv \int_{0}^{2 \pi} \xi_{0}^{\prime} \xi_{1}^{\prime \prime} \mathrm{d} \tau=-\int_{0}^{2 \pi} \xi_{0}^{\prime \prime} \xi_{1}^{\prime} \mathrm{d} \tau=\int_{0}^{2 \pi} \xi_{0}^{\prime \prime \prime} \xi_{1} \mathrm{~d} \tau .
$$

We will investigate the integral (10) in (9) in detail for a few key cases. Fig. 3 shows the time-dependence of each of the factors of the integrand in (10), i.e. $\xi_{0}$ and $\xi_{0}^{\prime \prime \prime}$ as well as a few different optimal damper motions, which we will explore below.

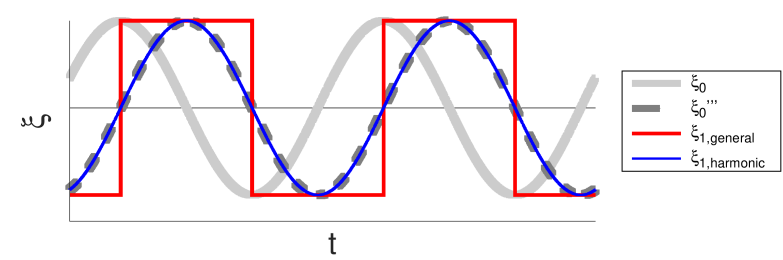

Figure 3: Time-dependence of each of the factors of the integrand in (10), i.e. $\xi_{0}$ (solid grey curve) and $\xi_{0}^{\prime \prime \prime}$ (dashed grey curve) as well as a few different optimal damper motions: The optimal general damper motion $\xi_{1, \text { general }}$ (red curve), see Sec. 2.1, and the optimal harmonic damper motion $\xi_{1, \text { harmonic }}$ (blue curve), see Sec. 2.2.

\subsection{General damper motion}

We first consider the case where the damper motion is unconstrained except for the space restriction $-a_{1} \leq$ $x_{1} \leq a_{1}$, i.e. $-1 \leq \xi_{1} \leq 1$. This could e.g. describe an ideal active damper, where any restrictions on the actuator power are neglected.

Clearly, the maximum value of the integral (10) over $\xi_{0}^{\prime \prime \prime} \cdot \xi_{1}$ is found by letting

$$
\xi_{1} \equiv \operatorname{sign}\left(\xi_{0}^{\prime \prime \prime}\right)=\operatorname{sign}(\sin \tau),
$$

which maximizes the integrand at all times, see Fig. 3. This trajectory requires the damper mass to impulsively jump between $\xi_{1}=-1$ and $\xi_{1}=+1$, which would require infinite available forces and power. While this is clearly not possible, (11) can serve as an approximation to the behaviour of real systems. Now, (10) gives

$$
I=\int_{0}^{2 \pi} \sin \tau \operatorname{sign}(\sin \tau) \mathrm{d} \tau=2 \int_{0}^{\pi} \sin \tau \mathrm{d} \tau=4,
$$

and we can state

Main result: The optimal motion of any mass damper, active or not, is obtained by letting the mass jump suddenly between its two outer positions at the time where the structure velocity changes sign, see Fig. 4.

The damping of any mass damper can be written

$$
\delta_{\max }=K \cdot 4 \mu \alpha,
$$

where $\mu=m_{1} / m_{0}$ is the damper mass ratio, and $\alpha=a_{1} / a_{0}$ is the damper amplitude ratio, and $0 \leq K \leq 1$ is an efficiency parameter.

We can gain further understanding into the energy loss mechanism of the ideal mass damper (13) by investigating the behaviour of a decaying structure with a 


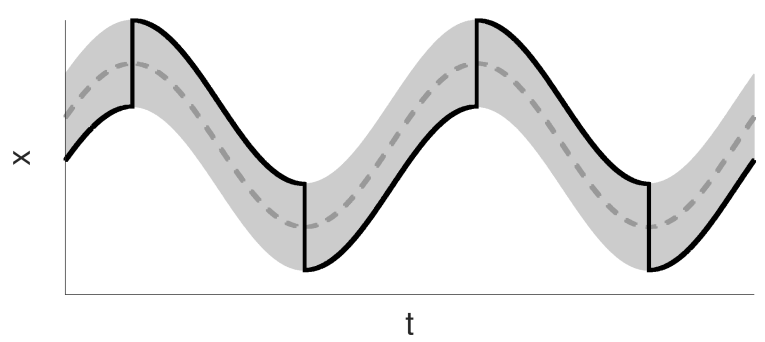

Figure 4: Illustration of the motion of the structure $x_{0}(t)$ (dashed grey curve), the available damper space (grey area), and the damper motion (black curve), in the case of an optimal mass damper, see (13). The damper starts with $\xi_{1}=-1$ until the structure reaches its maximum position $\xi_{0}=+1$, at which time $\xi_{1}$ jumps impulsively to $\xi_{1}=+1$. Two jumps in every oscillation cycle.

damper moving impulsively according to (11). An example is shown in Fig. 5. Each time the structure motion is at a turning point, $\dot{x}_{0}=0$, the damper impulsively moves relative to $m_{0}$ by a distance $\Delta x_{1}=2 a_{1} \operatorname{sign}\left(x_{0}\right)$ in the direction of $x_{0}$. The centre of mass at the position $\left(m_{0} x_{0}+m_{1} x_{1}\right) /\left(m_{0}+m_{1}\right)$ remains unaffected by internal forces, in particular of the damper motion, so the structure must move inwards with $\Delta x_{0}=-\mu \Delta x_{1}$. This decreases the amplitude by a factor

$$
\frac{x_{0}+\Delta x_{0}}{x_{0}}=\frac{a_{0}-2 \mu a_{1}}{a_{0}}=1-2 \mu \alpha .
$$

This happens twice during each oscillation cycle, so the exact logarithmic decrement due to the damper motion illustrated in Fig. 5 is

$$
\delta_{\text {decay }}=-2 \log (1-2 \mu \alpha) \approx 4 \mu \alpha .
$$

The final result, valid to first order in $\mu \alpha$, is identical to (13).

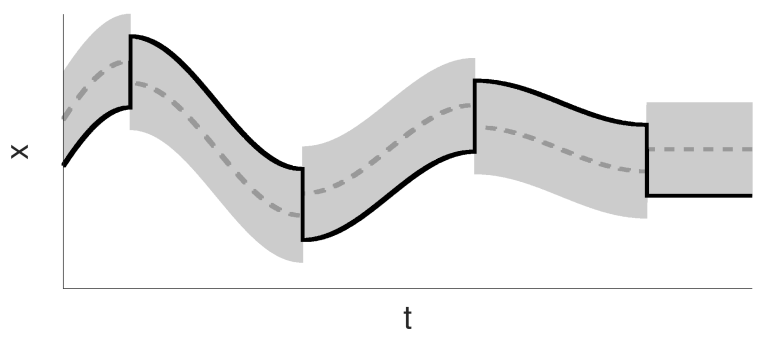

Figure 5: Illustration of the mechanism of damping for the system shown in Fig. 4. For each jump of $x_{1}, x_{0}$ impulsively moves in the opposite direction, so that the center-of-mass remains stationary. The potential energy associated with $x_{0}$ thus decreases. Observe the fixed size jumps of $x_{0}$, corresponding to still larger damping values for decreasing structure amplitudes. In the example shown, the structure comes to a complete stop after 4 damper movements.

\subsection{Harmonic damper motion}

We now turn to harmonically moving damper masses, e.g. in linear passive TMD devices. Consider a damper motion which is delayed from the structure motion by a constant phase angle $\varphi$ :

$$
x_{1}=a_{1} \cos (\omega t-\varphi)
$$

i.e.

$$
\begin{aligned}
\xi_{1} & =\cos (\tau-\varphi) \\
& =\cos (\varphi) \cos (\tau)+\sin (\varphi) \sin (\tau) .
\end{aligned}
$$

We then write

$$
\begin{aligned}
I & =\int_{0}^{2 \pi} \sin (\tau) \cdot(\cos (\varphi) \cos (\tau)+\sin (\varphi) \sin (\tau)) \mathrm{d} \tau \\
& =\cos (\varphi) \underbrace{\int_{0}^{2 \pi} \cos (\tau) \sin (\tau) \mathrm{d} \tau}_{=0}+\sin (\varphi) \underbrace{\int_{0}^{2 \pi} \sin ^{2}(\tau) \mathrm{d} \tau}_{=\pi} \\
& =\pi \sin (\varphi) .
\end{aligned}
$$

So we have $I=\pi$ for the optimal phase-lag $\varphi=\pi / 2$ (this phase-lag is well-known in the TMD litterature), see Fig. 3. In summary:

Secondary Result: The optimal harmonically moving mass damper lags behind the structure by $\varphi=\frac{\pi}{2}$, and obeys (13) with

$$
K=\frac{\pi}{4}
$$

We can gain further understanding of the above result by observing that the force from the damper moving with $x_{1}=a_{1} \sin \omega t$ is $F=-m_{1}\left(\ddot{x}_{1}+\ddot{x}_{0}\right)$. Ignoring the second term, which only gives a small (of order $\mu$ ) correction to the effective structure mass, we observe that

$$
F=m_{1} a_{1} \omega^{2} \sin \omega t=-m_{1} \frac{a_{1}}{a_{0}} \omega \dot{x}_{0}
$$

where we have used that $\dot{x}_{0}=a_{0} \omega \sin \omega t$. We observe that the damper reaction force in (20) is proportional to the structure motion, so the damper is equivalent to a dashpot with damping rate $c=m_{1} \alpha \omega$. The corresponding damping ratio is found as

$$
\zeta=\frac{c}{2 m_{0} \omega}=\frac{1}{2} \mu \alpha
$$

Using the well-known approximation $\delta \approx 2 \pi \zeta$ (excellent for values of $\zeta$ ), we recover (19).

\subsection{Connection to classical TMD theory}

We briefly turn to the more traditional linear system considerations normally applied to TMDs, see e.g. [1, 7]. We might call the system analyzed in the traditional approach the amplification-limited regime in contrast to the amplitude-limited regime considered above and described by our Results (13) and (19).

In the amplification-limited regime, TMDs are wellknown to be very efficient at low mass ratios, i.e. with

$$
\delta_{T M D \text { opt. }} \approx 2.2 \cdot \sqrt{\mu},
$$




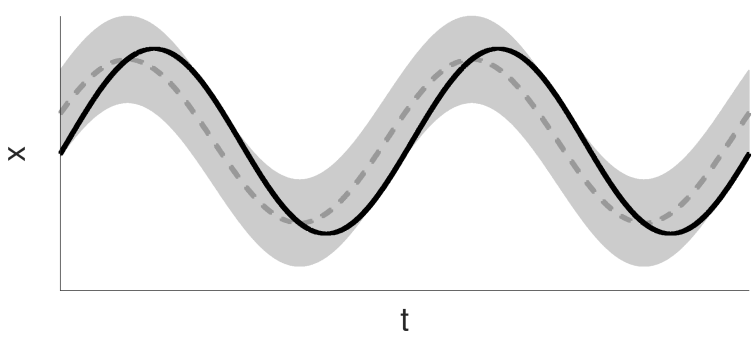

Figure 6: Motion of the structure and an optimal harmonically moving damper, see (19). Compare to Fig. 4.

see e.g. [9] (Table 1, case 1). This is in contrast the proportional dependence of $\delta$ on $\mu$ seen in the amplificationlimited regime, (13) and (19). However, the classically optimized TMD achieves this high efficiency for small mass ratios by greatly amplifying the motion of the damper mass.

Using [7], we note that an optimal TMD has the internal damping $\zeta_{1} \approx \sqrt{\frac{\mu}{2}}$, so the resonant peak of the damper response spectrum will occur with approximately

$$
\alpha=\frac{a_{1}}{a_{0}}=\frac{1}{2 \zeta_{1}}=\frac{1}{\sqrt{2 \mu}},
$$

so that (19) gives

$$
\delta=\pi \alpha \mu=2.2 \cdot \sqrt{\mu}
$$

in good agreement with (22). It should be emphasized that the damping must always obey

$$
\delta \lesssim \min \left(\delta_{\max }, \delta_{T M D} \text { opt. }\right),
$$

and that neither criteria (13) and (22) guarantee a certain level of damping. Rather, the criteria reflect the maximal damping that can be achieved.

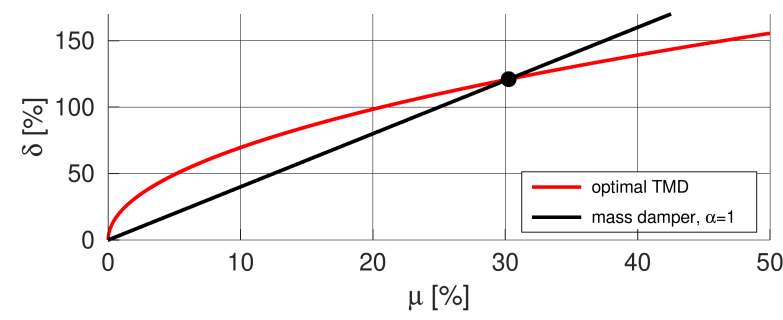

Figure 7: Comparison of performance of amplitude-limited and amplification-limited mass dampers. The red curve shows the damping due to an optimal TMD (without amplitude limitations), (22). The black curve shows the maximum damping with a fixed amplitude ratio $\alpha$, (13). In this example (with $\alpha=1$ ), the finite amplitude sets the limit (25) as long as $\mu<(2.2 / 4)^{2}=30 \%$, as can be seen by comparing (22) and (13).

\section{Examples}

\subsection{Method}

We now present a few examples of mass dampers constrained to a finite damper amplitude. We consider the damper motion as described by a linear equation of motion with a stiffness $m_{1} \omega_{1}^{2}$ and damping rate $2 m_{1} \omega_{1} \zeta_{1}$, until the amplitude of constraint $\left|x_{1}\right|=a_{1}$, where absorbing boundaries are employed. In order to make the fewest possible number of assumptions, we consider a simple form of end stops, which are ideally completely inelastic, i.e. absorbing all relative kinetic energy at the time of impact. This can be simulated using the interaction force $F_{i a}=F_{i a}\left(x_{1}, \dot{x}_{1}\right)^{2}$. The motion of the structure and the damper can be described by the ODE system

$$
\begin{aligned}
(1+\mu) \ddot{x}_{0}+\mu \ddot{x}_{1}+x_{0} & =-F_{i a}, \\
\ddot{x}_{0}+\ddot{x}_{1}+2 \omega_{1} \zeta_{1} \dot{x}_{1}+\omega_{1}^{2} x_{1} & =F_{i a},
\end{aligned}
$$

where

$$
F_{i a}=-F_{i} \operatorname{sign}\left(\xi_{1}\right) G\left(\frac{\left|\xi_{1}-1\right|}{b_{1}}\right) G\left(\frac{\dot{\xi}_{1} \operatorname{sign}\left(\xi_{1}\right)}{b_{2}}\right) .
$$

Here we have used the smoothed step function $G(y) \equiv \frac{1}{2}(1+\tanh (y))$ and the normalization constants $b_{1}$ and $b_{2}$, which have to be small in order to approximate the desired instantaneous inelastic collision, while not making the ODE system excessively stiff. Similarly, a suitable large impact force magnitude $F_{i}$ has been selected.

Below, we present results using two methods of analysis:

Method 1) Decay tests of the full system (26-27) started from a given amplitude, where $\delta$ is then computed directly from (6).

Method 2) Forced motion tests at a chosen amplitude $a_{0}$. Here $x_{0}=a_{0} \cos t$ is given, and (27) is solved until a stationary periodic solution has been found. Then, the energy dissipated during one cycle is computed using (8) and (10), and $\delta$ is then found by (7).

The two methods agree in many scenarios, but significant deviations can arise for 2DOF system (such as the structure + damper system), as we shall see below. Experimentally, it is often easier to perform decay tests (Method 1), while harmonically forced tests (see Method 2) can be more challenging to carry out. However, one must be very careful when using one kind of test in place of the other. In particular, Vortex Induced Vibrations (VIV) arise due to a periodic forcing, which is more closely related to the damping measured by harmonically forced tests, and it is risky to then measure the damping only by decay tests, in particular for 2DOF systems such as structures with a mass damper, see e.g. [13]. It is generally recommended, that both types of forcing are analyzed, at least numerically, when a structure+damper system is designed.

\subsection{Tuned mass damper (TMD)}

We first consider a couple of examples of TMDs with $\mu=3 \%$, see Fig. 8. The damper parameters are given

\footnotetext{
${ }^{2}$ alternatively, the absorbing end-stops can be simulated accurately and efficiently by a punctuated-time scheme
} 
relative to the classical Hartog optimum [1], [9], with $\omega_{1, \text { opt. }}=\frac{1}{1+\mu}$ and $\zeta_{1}^{2}=\frac{3}{8} \frac{\mu}{1+\mu}$.

The system behaviour is simulated using (26-27), and the damping is shown based on both methods described above in Sec. 3.1.

Fig. 8 shows the damping as a function of the amplitude (normalized with the maximum damper amplitude) for three different tunings relative to $\omega_{1, \text { opt. }}$ : An under-tuned TMD $\omega_{1}=0.8 \cdot \omega_{1, o p t}$, an optimally tuned TMD $\omega_{1}=\omega_{1, o p t .}$, and an over-tuned TMD $\omega_{1}=1.2 \cdot \omega_{1, \text { opt. }}$.

For small amplitudes, the system dynamics is linear (the amplification-limited regime, see Sec. 2.3), and the harmonically forced test damping is a constant for each tuning. The decay test damping is observed to fluctuate erratically in some cases, with occasional measurements of $\delta<0$, illustrating the difficulty of describing some 2DOF systems by decay tests. Furthermore, the damping can be higher in decay or in forced motion, depending on the specific tuning, highlighting the need for simulations appropriate to the specific application.

For larger amplitudes, (the amplitude-limited regime) the damping follows $(13,19)$ to a good approximation. Observe that the damper tuning appears less important in this regime. It does seem that the softer tuning gives a slightly higher damping for large amplitudes, suggesting that the amplitude is restricted by the stiff spring for $\omega_{1} \gtrsim \omega_{1, o p t .}$.

Observe that the damper performance approaches the optimal value (19) the optimal and under-tuned cases, when the harmonically moving damper utilizes all the available space, i.e. the red curve touches the dashed grey curve in Fig. 8 (center panel).

\subsection{Free mass with inelastic end-stops}

We also present the damping as a function of structure amplitude for a free mass with inelastic end-stops (incidentally the same system that was shown in Fig. 2). Technically speaking, we solve (26-27) with $\omega_{1}=\zeta=0$ using both methods described above in Sec. 3.1.

The results are shown in Fig. 9 (top panel). Observe that a damping exceeding the maximum performace of a harmonically moving damper (19) is observed for a large range of structure amplitudes. As one might expect, the damping becomes very low for small amplitudes, where the damper might float inside the structure without frequent interations between the two. Fig. 9 (bottom panel) shows an example of a decaying system. For purposes of illustration, the mass ratio is chosen rather high, $\mu=20 \%$. The amplitude is observed to initially decay almost linearly with time, which is characteristic for damping with $\delta \sim 1 / a_{0}$, see (13). After the structure has decayed to an amplitude $a_{0} \approx a_{1}$, the damper drifts slowly, with infrequent interactions with the structure. This corresponds to the irregular damping behaviour shown in the top frame of Fig. 9.
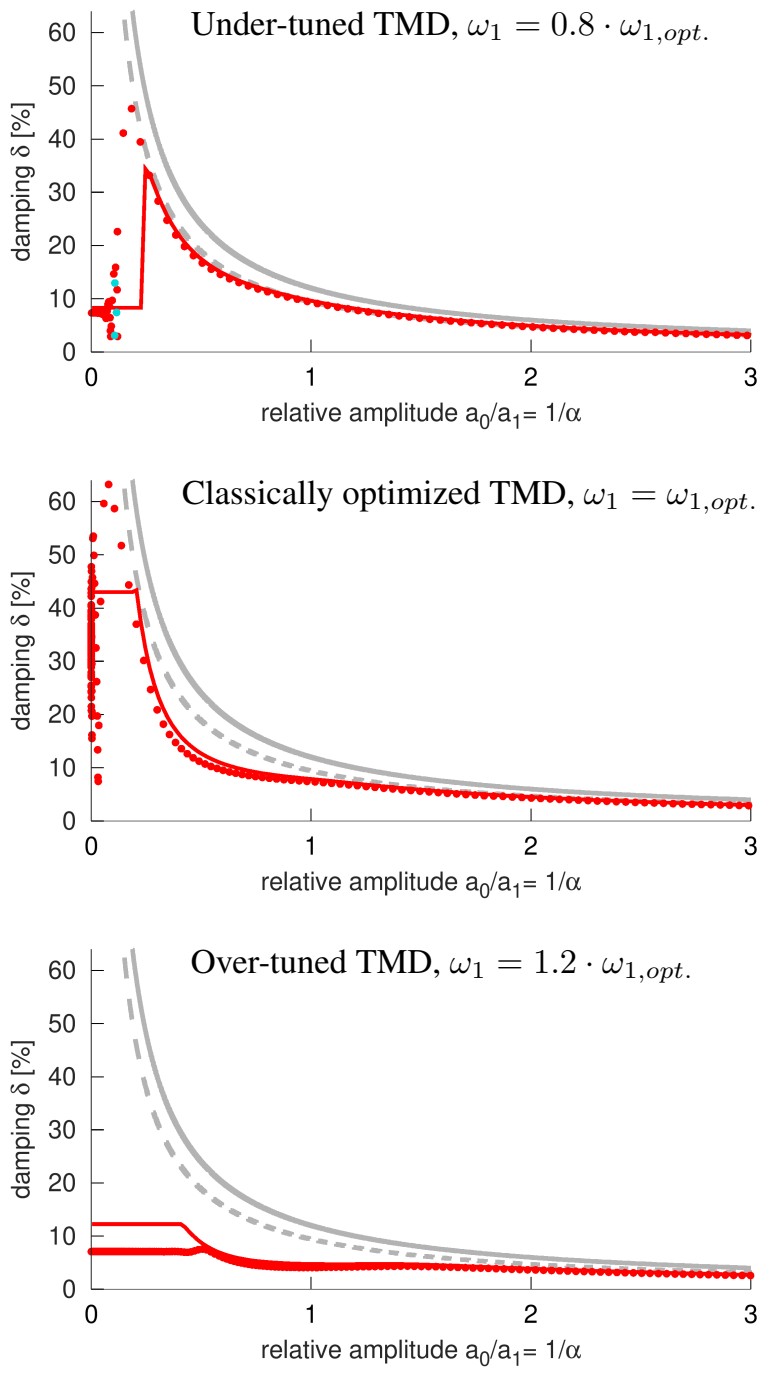

Figure 8: Damping as a function of the structure vibration amplitude $a_{0}$ (normalized by the maximum damper amplitude $a_{1}$ ) for a TMD with $\mu=m_{1} / m_{0}=3 \%$, see Sec. 3.2. The red curve shows damping as computed from (27) by method 2, see Sec. 3.1. The red dots show the damping as measured from a simulated decay test (26-27) using method 1, see Sec. 3.1.

Observe the erratic behaviour observed for small amplitudes in some cases, corresponding to a more complicated decay, e.g. a beating of 2 modes. The cyan dots indicate negative recorded values of $\delta$.

The solid grey curve is the maximum damping for any mass damper (13). The dashed grey curve is the maximum damper for a harmonically moving mass damper, (19). Each panel shows a different tuning for a mass damper with $\mu=m_{1} / m_{0}=3 \%$. See also Sec. 3.2, and compare to Fig. 9.

\section{Conclusions}

We have found that general mass dampers in the very common amplitude-limited regime obey a very simple rule, that the damping cannot exceed a critical value $\delta_{\max }$. The result, (13), can be interpreted as a prediction of the 

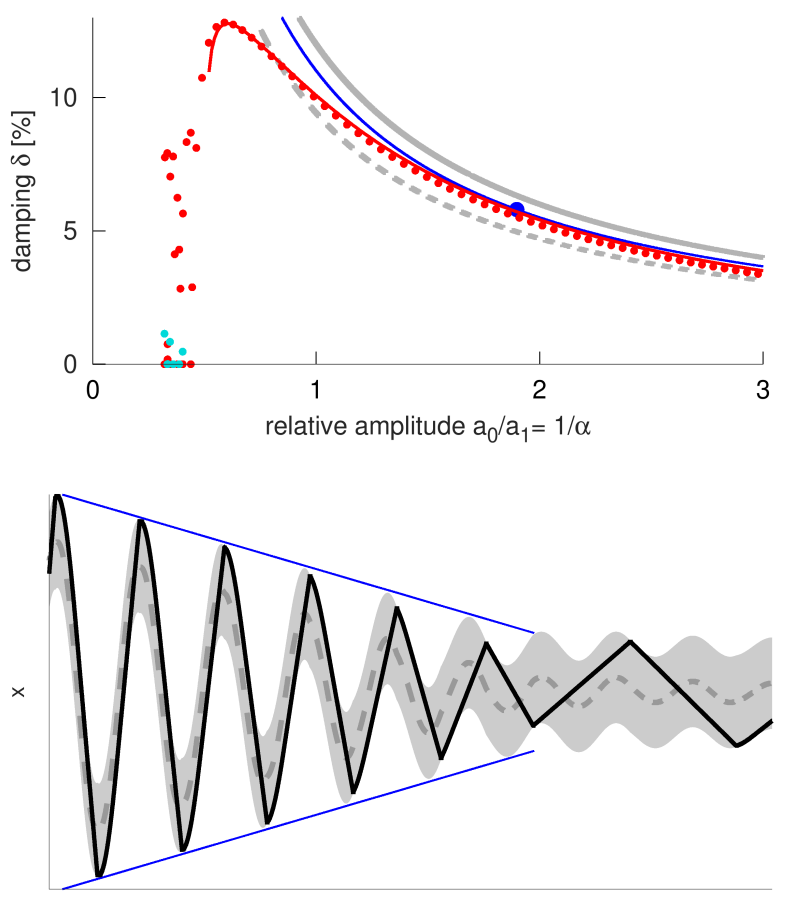

$\tau$

Figure 9: Damping as a function of $a_{0}$ for a free mass with inelastic end-stops, see Sec. 3.3.

Top panel: Damping computed as described in Sec. 3.1, with $\mu=m_{1} / m_{0}=3 \%$. See also the caption of Fig. 8. The blue curve and the blue dot corresponds to the maximal value of $I$, which we briefly note occurs at $I(\alpha=0.53)=3.7$.

Bottom panel: Example of the motion of the structure and the damper for a rather heavy ( $\mu=20 \%$ ) free damper with inealistic end-stops. The characteristic behaviour of the amplitude decreasing nearly linearly with time is indicated by the blue lines. At low amplitudes, the damping is small, and collisions occur only occasionally. Compare to Figs. 4 and 5.

actual damping, if one uses a reasonable value of the pre-factor $K$ in (13) based on simulations and experience with mass dampers.

The result has been discussed in connection with Tuned Mass Dampers (TMDs), see (19), which have typically in the litterature been considered as linear systems, in what we denote the amplification-limited regime. Our results can be interpreted as relating the required damping to the required amplification of a TMD.

Our general result also sets limits on the maximum attainable damping from active dampers. In particular, an active damper with unlimited force and power can in principle obtain the ideal result (13) with $K=1$. In contrast, optimal TMDs are observed to obtain a behaviour close to the ideal harmonic motion case (19) with $K=\frac{\pi}{4}$ when the optimal TMD uses the full available space. This means that the added benefit of using an active damper as opposed to an optimal TMD is limited, as long as the motion is harmonic. Rather, the benefit of using active or semi-active dampers are found in the response to other kinds of forcing, e.g. the ability of an active damper to counteract motions of varying frequencies as well as transient motions.

We have presented results of simulations, where the damping from both TMDs and other mass dampers is described, both in terms of decay tests and in terms of harmonically forced tests. The two methods give very similar results in the amplitude-limited regime. In the amplification-limited regime, decay tests are difficulet to interpret, indicating that one needs to be very careful when measuring damping of 2DOF systems based solely on decay tests.

\section{Outlook}

We propose that researchers and engineers generalize the results presented above to a full spectral analysis including the $H_{\infty}$ characterization of the damping (see [13]), which we have considered beyond our current scope. Furthermore an analysis of liquid / sloshing dampers in relation to the above results could be of significant interest.

It is our hope that our results, in particular (13), may be used in the practical selection and design of mass dampers, and that it may encourage increased focus on the amplitude limits of mass dampers.

\section{References}

[1] Den Hartog, J.P. Mechanical Vibrations, 4th. ed.; Dover reprint, 1985 (1956)

[2] EUROCODE 1-4:Wind Actions, EN1991-1-4 (1991).

[3] Christian Petersen, Schwingungsdämpfer im Ingenieurbau, Maurer \& Söhne GmbH \& Co. KG, München (2001)

[4] G. W. Housner et al., Structural Control: Past, Present, and Future, J. Eng. Mech 123 (9), pp. 897 971, (1997)

[5] de Silva, C.W. ,Vibration Damping, Control and Design, CRC Press, Taylor \& Francis Group LLC (2007)

[6] D.J. Inman, Engineering vibration, Prentice-Hall, (2014)

[7] Krenk, S. Frequency Analysis of the Tuned Mass Damper, Trans. ASME (72), pp. 936-942 (2005)

[8] Tophøj, L. \& Grathwol, N. \& Hansen, S.O., Effective Mass of Tuned Mass Dampers, MDPI Vibrations 1(1), pp. 192-206 (2018)

[9] G.B. Warburton, Optimum Absorber Parameters for Various Combinations of Response and Excitation Parameters, Earthq. Eng. Struct. Dyn. 10, pp. 381401 (1982)

[10] Elias, S.; Matsagar, V. Research developments in vibration control of structures using passive TMDs. Annu. Rev. Ctrl., 44, 129-156 (2017)

[11] Weisner, K.B. Tuned mass dampers to reduce building wind motion. In ASCE Convention and Exposition, Preprint 3510, NY, USA (1979) 
Laust Tophøj \& Nikolaj Grathwol, Mass Dampers with Limited Amplitude, Preprint, November 18, 2021

[12] Fujimo, Y.; Abé, M. Design formulas for tuned mass dampers based on a perturbation technique. Earthq. Eng. Struct. Dyn., 22, 833-854 (1993)

[13] Asami, T.,Optimal Design of Double-Mass Dynamic Vibration Absorbers Arranged in Series or in Parallel, J. Vib. Acoust., 139(1): 011015 (2017)

[14] Brodersen, M.L., Bjørke, A-S., \& Høgsberg, J.B., Active tuned mass damper for damping of offshore wind turbine vibrations. Wind Energy, 20(5), pp. 783-796, (2017)

[15] Alkhatib, R. \& Golnaraghi, M.F.,Active Structural Vibration Control: A Review, Shock and Vib. Digest 35, 5, pp. 367-383, (2003)

[16] Thomas, M.D. \& Sadek, M.M. The Effectiveness of the Impact Damper with a Spring-Supported Auxiliary Mass, J. Mech. Eng. Sci. 16, pp. 109-116, (1974)

[17] Yang, MY, Koopmann, GH, Lesieutre, GA, \& Hambric, SA., Attenuation of High Amplitude Vibrations With Particle Dampers, Proc. ASME, Congress: Noise Control and Acoustics. New Orleans, LA, USA., pp. 113-118 (2002)

[18] Duncan, M.R., Wassgren, C.R. \& Krousgrill, C.M., The damping performance of a single particle impact damper, J. Sound Vib. 286 (1-2), pp. 123-144, (2005)

[19] Hunt, J.B. , Dynamic Vibration Absorbers, Mechanical Engineering Publications (1979)

[20] Ema, S. \& Marui, E., A fundamental study on impact dampers, Int. J. Mach. Tools Manufacture 34 (3), pp. 407-421, (1994)

[21] Okolewska B.B., Analysis of an impact damper of vibrations, Chaos, Solitons \& Fractals 12 (11), (2000)

[22] Gagnon, L., Morandini, M. \& Ghiringhelli, G.L., A review of particle damping modeling and testing, $\mathrm{J}$ Sound Vib. 459, 114865, (2019)

[23] Lu, Z., Wang, Z., Masri, SF. \& Lu, X., Particle impact dampers: Past, present, and future. Struct Control Health Monit. (2018)

[24] Shaw, S.W., System Having Rigid Amplitude Constraints, Parts 1-2, J. Appl. Mech. Jun 1985, 52(2), pp. 453-458 and 459-464. (1985)

[25] Masri, S. F., and Caughey, T. K. On the Stability of the Impact Damper, ASME. J. Appl. Mech. 33(3): pp. 586-592, (1966)

[26] Petit, F., Exploring the limitations of linear and nonlinear vibration absorbers, $\mathrm{PhD}$ thesis, Ghent University. Faculty of Engineering and Architecture, Ghent, Belgium, (2012)

[27] Reed, D. \& Yu, J. \& Yeh, H. \& Gardarsson, S., Investigation of Tuned Liquid Dampers under Large Amplitude Excitation. J.Eng.Mech. ASCE 124 (1998)
[28] Ibrahim, R.A. Liquid Sloshing Dynamics, Theory and Applications; Cambridge University Press: Cambridge, UK (2005)

[29] Hochrainer, M.J.; Adam, C.; Ziegler, F. Application of tuned liquid column dampers for passive structural control. In Proc. ICSV 7, GarmischPartenkirehen, Germany, 4-7 July 2000. 